\title{
Miocardiopatía Takotsubo postparto: parto distócico como posible factor desencadenante
}

\author{
Zoraida Frías Sánchez ${ }^{1}$, Manuel Pantoja Garrido², Guillermo Gómiz Rodríguez², \\ Francisco Javier Pantoja Rosso. ${ }^{2}$ \\ ${ }^{1}$ Unidad de Gestión Clínica de Obstetricia y Ginecología del Hospital Universitario Virgen Macarena de Sevilla. ${ }^{2}$ Servicio \\ de Ginecología y Obstetricia del Hospital General Santa María del Puerto, Puerto de Santa María, Cádiz. España.
}

\section{RESUMEN}

La miocardiopatía Takotsubo, o miocardiopatía por estrés, se define como una disfunción del segmento apical del ventrículo izquierdo, aguda, transitoria y reversible. Normalmente se relaciona con algún tipo de agente estresante, ya sea físico o emocional, pudiendo confundirse este cuadro con un síndrome coronario agudo. Se considera una patología rara, pero que es importante incluir en el diagnóstico diferencial de los trastornos cardiacos, sospechándolo previamente por la sintomatología. Está caracterizado por alteraciones en el electrocardiograma, como elevación del segmento ST en derivaciones precordiales e inversión de la onda T, elevación de las enzimas de necrosis miocárdica o patrones alterados en la ecocardiografía, como la disfunción ventricular apical con hiperquinesia de zonas basales. En cuanto a la fisiopatología, parece que la estimulación simpática juega un papel importante, en el que la disfunción ventricular se desencadena por algún tipo de estrés físico o emocional. Éste provoca una descarga de catecolaminas con un efecto cardiotóxico debido, en su mayor parte, al efecto del adenosín monofosfato cíclico. El tratamiento consiste en las medidas soporte de una miocardiopatía, evitando los fármacos teratogénicos o perjudiciales durante el periodo de lactancia. La evolución es favorable en la mayoría de los casos y el cuadro remite por completo, por lo que el pronóstico en líneas generales se considera bueno. En este trabajo se expone el caso de un parto instrumentado, en el que se manifiesta un cuadro compatible con una miocardiopatía Takotsubo, posiblemente relacionado con un procedimiento invasivo como factor desencadenante.

\section{PALABRAS CLAVE: Miocardiopatías, cardiomiopatía Takotsubo, síndrome de balón apical, miocardiopatía por estrés}

\section{SUMMARY}

Takotsubo cardiomyopathy or stress cardiomyopathy is defined as an acute, transient, and reversible dysfunction of the apical segment of the left ventricle. It is usually associated with some sort of stressor, whether physical or emotional, and could be confused with an acute coronary syndrome. It is considered a rare disease, so it is important to include it in the differential diagnosis, previously suspecting the symptoms. It is characterized by alterations in the electrocardiogram such as ST segment elevation and inversion of the T wave in precordial leads, elevation of myocardial necrosis enzymes, or altered patterns on echocardiography such as apical ventricular dysfunction with hyperkinesia of basal areas. Regarding the pathophysiology, it seems that sympathetic stimulation plays an important role, in which ventricular dysfunction is triggered by some kind of physical or emotional stress. This causes a release of catecholamines with cardiotoxic effects due, mostly, to the effect of cyclic AMP. Treatment involves cardiomyopathy supportive care, avoiding teratogenic or harmful drugs during lactation. The evolution is favorable in most cases and the clinical 
picture abates completely, so the prognosis is generally considered good. In this paper, we expose a case of an instrumental labor in which a clinical picture compatible with Takotsubo cardiomyopathy is presented, triggered by an invasive procedure.

\section{KEY WORDS: Myocardiopathies, Takotsubo cardiomyopathy, apical ballooning syndrome, stress cardiomyopathy}

\section{INTRODUCCIÓN}

La miocardiopatía Takotsubo se considera una de las variantes peculiares de la miocardiopatía postparto. Es un síndrome adquirido que se caracteriza por una disfunción transitoria y reversible de la región apical del ventrículo izquierdo, pudiendo confundirse dicho cuadro con un síndrome coronario agudo $(1,2)$. La incidencia de esta patología se da fundamentalmente, en mujeres postmenopáusicas, entre 60 y 70 años, siendo desencadenado por cirugías u otras causas estresantes que conllevan un aumento de los niveles de catecolaminas plasmáticos (2). Tanto la etiología como la fisiopatología son temas actualmente poco conocidos, teniendo como uno de los pilares etiopatogénicos más importantes, la activación simpática. Ésta es responsable de la liberación excesiva de catecolaminas que provocan una disfunción microvascular $y$, como consecuencia, una cardiotoxicidad inmediata (1).

La clínica se manifiesta como dolor torácico, disnea, ortopnea (3) o, incluso en ocasiones, puede pasar desapercibida o confundirse con un síndrome coronario agudo $(1,2)$. En cuanto al diagnóstico, a parte de la sospecha clínica previa, nos podemos basar en los hallazgos electrocardiográficos presentes en un alto porcentaje de las ocasiones. Esto justifica la elevación del segmento ST en derivaciones precordiales $(1,2)$, o la inversión de la onda T en casi un $97 \%$ de los pacientes (4). En la ecocardiografía, la anomalía más común es el "balonamiento apical" $(1,5)$ del ventrículo izquierdo, como resultado de una hipomotilidad del ápex, acompañada de una hiperquinesia del resto de segmentos ventriculares (5).

El tratamiento debe individualizarse, ya que irá en función de las características de su cuadro $(5,6)$. El pronóstico, por lo general, es muy bueno, ya que se trata de un síndrome reversible (5). La mortalidad hospitalaria es inferior al 1\% (5) y el riesgo de recurrencia está aún por definir, ya que no existen datos suficientes para poder concluir sobre dicha cuestión $(7,8)$.

El objetivo de esta comunicación es presentar el caso de un parto instrumentado, en el que se manifiesta un cuadro compatible con una miocardiopatía Takotsubo, posible consecuencia del procedimiento obstétrico como factor desencadenante.

\section{Caso clínico}

Primigesta de 28 años, de origen caucásico, sin antecedentes cardiovasculares relevantes. La gestación discurre con absoluta normalidad, llevando un control acorde a un embarazo de bajo riesgo. No obstante, la paciente acude con frecuencia al Servicio de Urgencias narrando diferentes motivos de consulta, dándose de alta sin objetivarse patología en ninguna de las consultas. Destacamos un episodio de hipertensión arterial puntual relacionado con una situación de estrés personal, que se resuelve espontáneamente, sin necesidad de tratamiento. El resto del embarazo evoluciona favorablemente, llegando hasta la semana $40+2$ días, e ingresa por trabajo de parto. Previo ingreso, se realiza una ecografía abdominal, en la que se describe feto único en posición cefálica con el resto de hallazgos ecográficos normales. El registro cardiotocográfico fetal no presenta alteraciones y la exploración muestra que la paciente se encuentra en fase activa de parto. El periodo de dilatación transcurre sin incidencias, finalizando el parto con ventosa obstétrica por agotamiento materno. Durante la instrumentación, se avisa al Servicio de Anestesia por ansiedad materna y dolor insoportable pese a tener anestesia epidural. Finalmente, se realiza el parto, obteniéndose un recién nacido de 3565 gramos, sano.

En el control posparto, inicia cuadro de malestar general, sensación disneica y ortopnea que no remite. A la exploración, se observa una tensión arterial de $170 / 110 \mathrm{mmHg}$, taquicardia moderada de 115 latidos por minuto y edema bilateral que llega hasta el hueco poplíteo. En ese momento, se avisa al tocólogo de guardia, que descarta patología obstétrica, y contacta con el Servicio de Medicina Interna para valoración del cuadro. Ante la clínica de la paciente y el empeoramiento progresivo, la Unidad de Medicina Interna solicita un electrocardiograma, en el que se observa un supradesnivel del segmento ST en derivaciones precordiales (Figura 1), y una radiografía de tórax con imagen de cardiomegalia y derrame pleural bilateral (Figura 2). Con la sospecha clínica de insuficiencia cardíaca es derivada a la Unidad de Cuidados Intensivos del hospital de referencia. 


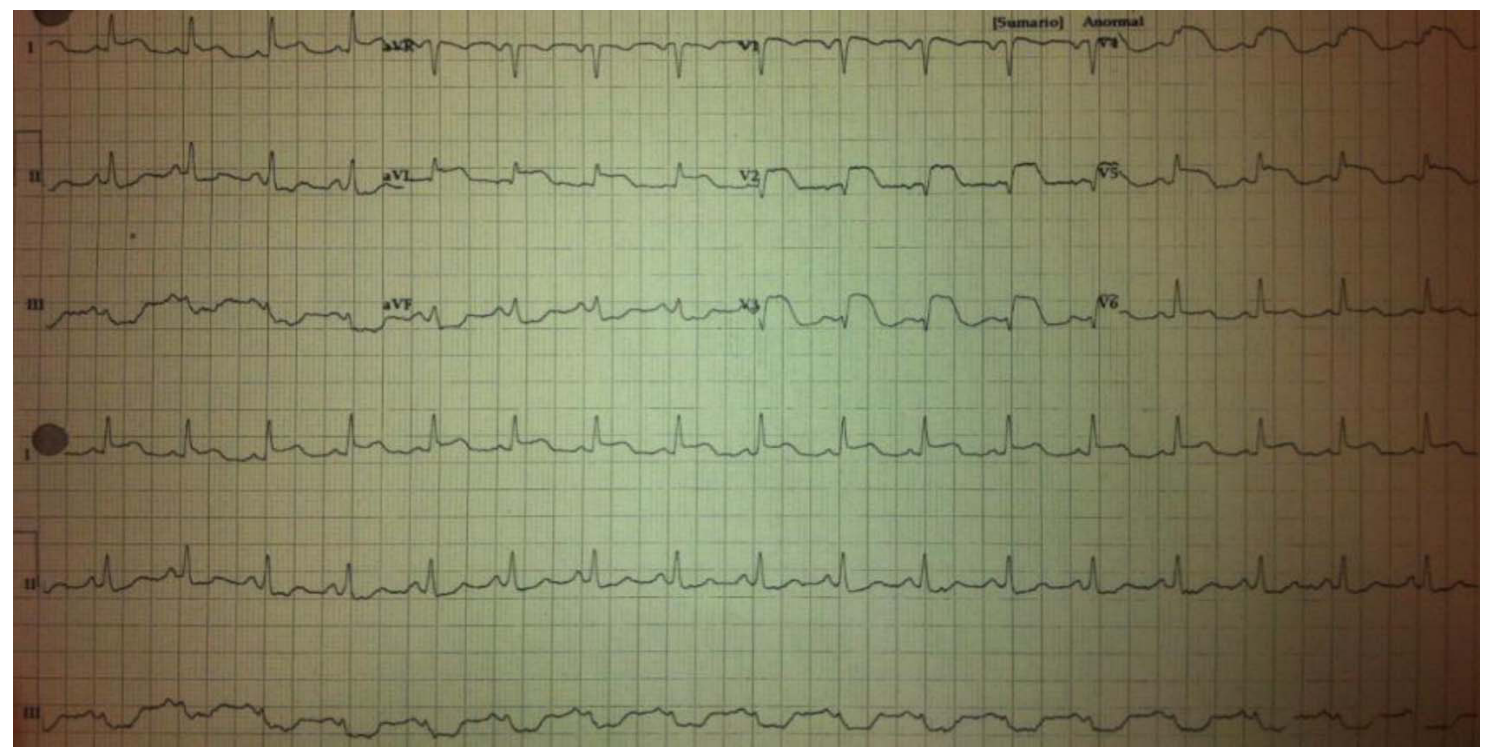

Figura 1. Electrocardiograma con supradesnivel del segmento ST en derivaciones precordiales.

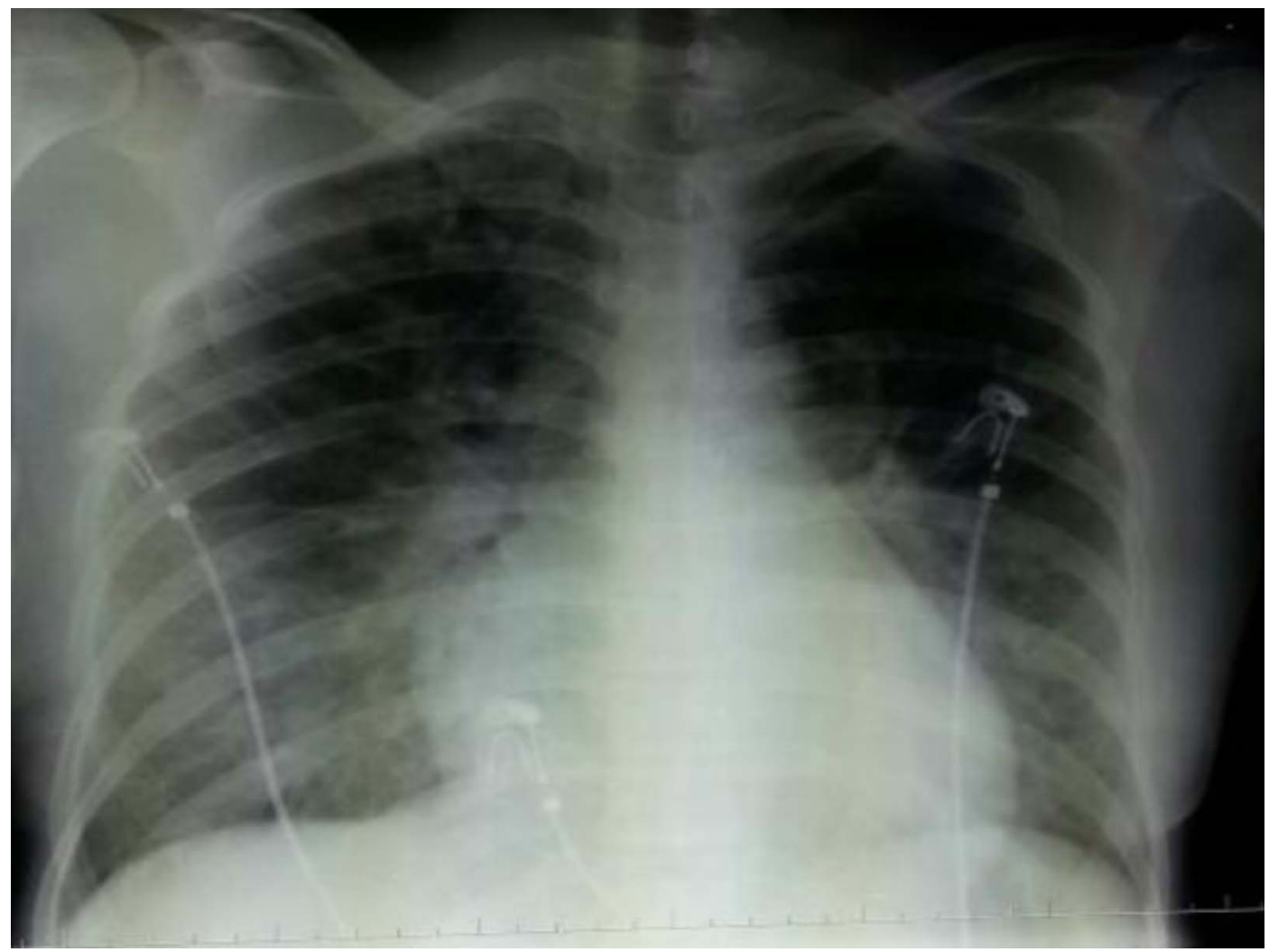

Figura 2. Radiografía de tórax con imagen de cardiomegalia y derrame pleural bilateral. 
La exploración física al llegar muestra una paciente con mal estado general, taquicardia de 115 latidos por minuto, taquipnea de 20 respiraciones por minuto, ortopnea a 45으, saturación de oxígeno en aire ambiente del $79 \%$ y una presión arterial de 170/105 mmHg. La auscultación cardiorrespiratoria se describe con crepitantes finos bilaterales e hipoventilación bibasal. La palpación abdominal no demuestra alteraciones llamativas, presentando edema bilateral con fóvea positiva en miembros inferiores. El estudio analítico reveló marcadores de necrosis miocárdica elevados (Troponina T 0,50 $\mathrm{ng} / \mathrm{ml}$ y creatinin fosfoquinasa fracción MB 17,5 ng/ $\mathrm{ml})$ y función hepática normal. La función renal, el ionograma, la calcemia, fosfatemia y la función tiroidea fueron normales. Ante dicha situación clínica, se solicita tomografía axial computarizada, gasometría arterial y se repite el electrocardiograma. La gasometría muestra hipoxemia con una presión de oxígeno de $30 \mathrm{mmHg}$ (pO2 $30 \mathrm{mmHg}$ ), en cambio, la tomografía axial describe un infiltrado de predominio lobar superior, entremezclado con un patrón alveolar difuso de localización parahiliar bilateral. Se asocia derrame pleural bilateral de predominio derecho y cardiomegalia a expensas de cavidades izquierdas. El electrocardiograma muestra ya, además del segmento ST elevado, inversión de la onda T en las derivaciones I y aVI (Figura 3). Dado que la situación actual evidencia un cuadro de disfunción ventricular, se solicita una ecocardiografía. Los hallazgos fueron los siguientes: ventrículo izquierdo (VI) dilatado con severa hipocontractilidad distal y apical con zonas de hiperquinesia en segmentos basales (fracción de eyección VI [FEVI]: 27\%) y derrame pericárdico ligero, con ausencia de valvulopatía. Por tanto, los datos clínicos, radiológicos y analíticos orientan el diagnóstico hacia una miocardiopatía por estrés (cardiomiopatía Takotsubo).

Ingresada en la Unidad de Cuidados Intensivos, se instaura tratamiento con depleción de volumen con el cual evoluciona favorablemente. Tras la estabilización de la paciente, se traslada a Medicina Interna, quien sigue su evolución y pauta tratamiento con diuréticos y vasodilatadores. La evolución es claramente satisfactoria con desaparición del infiltrado alveolar, confirmado por radiografía de tórax. La ecocardiografía de control muestra una afectación apical de la contractilidad con fracción de eyección en el límite de la normalidad. El alta hospitalaria es entregada a los seis días del ingreso con tratamiento domiciliario de carvedilol 6,25 mg cada 12 horas, furosemida $10 \mathrm{mg} /$ día, ramipril 5 $\mathrm{mg} / 12$ horas y atorvastatina $20 \mathrm{mg} / \mathrm{día}$.

En las consultas de seguimiento la paciente mantiene tensiones arteriales normales y pruebas complementarias dentro de la normalidad. El electrocardiograma de control presenta ritmo sinusal, con eje normal y sin alteraciones en la repolarización, y el ecocardiograma describe, como conclusión, una función sistólica recuperada y cavidades izquierdas no dilatadas. En las últimas visitas se encuentra asintomática y se retira la medicación, dándose finalmente de alta del Servicio por excelente evolución y remisión completa.

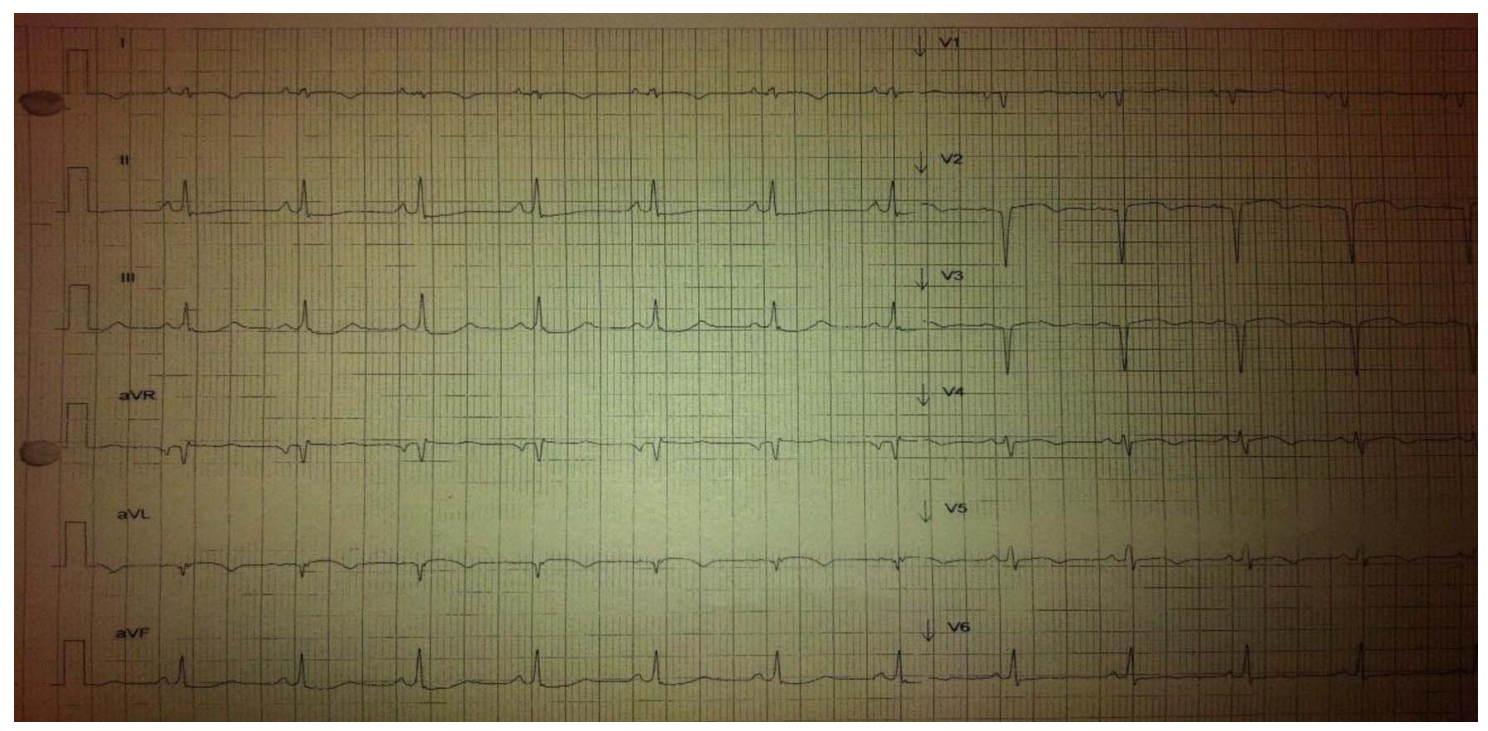

Figura 3. Electrocardiograma con ST elevado e inversión de la onda T en las derivaciones I y aVI. 


\section{DISCUSIÓN}

La miocardiopatía Takotsubo se considera una de las variantes peculiares de la miocardiopatía postparto, cuyo nombre es debido a la morfología típica en forma de "balón apical" que recuerda al tako-tsubo, recipiente usado en Japón para la pesca de pulpos (5). Fue descrita por primera vez por Dote y cols (9), al identificar una extensa acinesia en ápex sin lesiones coronarias significativas. La miocardiopatía por estrés es un síndrome adquirido que se caracteriza por una disfunción transitoria y reversible de la región apical del ventrículo izquierdo, pudiendo confundirse dicho cuadro con un síndrome coronario agudo $(1,2)$.

La incidencia de esta patología se da, fundamentalmente, en mujeres postmenopáusicas, de entre 60 y 70 años, siendo desencadenado por cirugías u otras causas estresantes que conllevan un aumento de los niveles de catecolaminas plasmáticos (2). La etiología no está definida con certeza y probablemente sea multifactorial (10). No obstante, se ha visto que la actividad simpática y el exceso de niveles de catecolaminas provocan una alteración a nivel de la microcirculación. Una de las teorías fisiopatológicas habla del posible efecto de la descarga catecolaminérgica en la pared vascular de las arterias coronarias, provocando, un espasmo de las mismas y la aparición de la sintomatología descrita. Otra de las teorías describe la posibilidad de que, en la zona apical, haya un aumento de los receptores o bien, una hipersensibilidad a catecolaminas, por lo que produciría una dilatación de dicha zona, dando la imagen típica de esta patología (10). Está en discusión la opción de que el aporte exógeno de catecolaminas en contexto de procedimientos intervencionistas anestésicos pueda ser el desencadenante del cuadro (9).

En nuestro caso, hablamos de una paciente joven, en edad fértil y sin comorbilidad, que presenta un parto distócico en el que se requiere anestesia locorregional en el momento de finalizarlo, por ansiedad materna y dolor intenso percibido como insoportable por la paciente. La clínica inicial se manifiesta como dolor torácico, disnea, ortopnea (3) e incluso, en ocasiones, puede pasar desapercibida o confundirse con un síndrome coronario agudo $(1,2)$. En la exploración física los pacientes graves pueden demostrar signos de fallo cardíaco severo, edema pulmonar agudo, hipotensión arterial o shock.

El electrocardiograma puede evidenciar intervalo PR prolongado, intervalo QT prolongado, elevación del segmento ST en derivaciones precordiales (típicamente en derivaciones V3 a V6), inversión profunda de la onda $T$ en múltiples derivaciones y ondas $Q$ patológicas en derivaciones anteroseptales y laterales (9). La prueba que confirma dicha entidad es la ecocardiografía, en la que se obser- va una hipercinesia de las zonas basales asociada a una hipocinesia o acinesia del ápex del ventrículo izquierdo. Nuestro caso clínico demuestra la existencia de esa morfología típica en la prueba, que nos orientó hacia la sospecha de una posible miocardiopatía. El tratamiento de la cardiomiopatía de Takotsubo requiere, generalmente, sólo medidas de apoyo durante la fase aguda (10). Se usan vasodilatadores, depleción de volumen y diuréticos como base terapéutica, así mismo, el uso de agentes inotrópicos está contraindicado durante la fase aguda ya que aumenta el riesgo de padecer arritmias cardiacas (10).

Las mujeres con esta patología presentan una rápida mejoría clínica y ecocardiográfica, y un excelente pronóstico (9). La serie más grande, la cual incluyó 88 pacientes, describe una mortalidad hospitalaria del 1\% (11). La recurrencia de miocardiopatía por estrés parece ser rara, aunque aún no está bien definida por falta de datos (7). Las complicaciones fundamentales son el edema pulmonar o el fallo cardiaco izquierdo (9). Finalmente, la paciente de nuestro caso tuvo una evolución excelente y una recuperación completa, con remisión de todas las alteraciones que habían aparecido durante el desarrollo del cuadro.

\section{CONCLUSIÓN}

La miocardiopatía Takotsubo o, también llamada, miocardiopatía por estrés es una entidad típica de mujeres postmenopaúsicas, con carácter reversible y transitorio, caracterizada por ausencia de estenosis importantes de las arterias coronarias. La base etiológica se encuentra en la hiperactividad simpática, la descarga de catecolaminas, la posterior disfunción microvascular y la consecuente cardiotoxicidad inmediata. El pronóstico, en general, es muy bueno y el cuadro clínico remite por completo en un periodo máximo de tres meses. No obstante, hay que tener en cuenta que las mujeres en el postparto inmediato representan un grupo susceptible de padecer dicho cuadro, variante peculiar de la miocardiopatía periparto. Por lo tanto, se considera esencial el diagnóstico precoz de esta entidad, ya que tanto el tratamiento como el pronóstico depende en gran medida del reconocimiento de la miocardiopatía.

\section{REFERENCIAS}

1. Russo M, Sappa R, Gianfagna E, Salame K, Sinagra $\mathrm{G}$, Proclemer A. Takotsubo and surroundings. Step by step postpartum stress-induced cardiomyopathy shows its facet. A peculiar case of atypical takotasubo in the peripartum period. G Ital Cardiol (Rome) 2014;15(1):51-5.

2. Minatoguchi M, Itakura A, Takagi E, Nishibayashi M, Kikuchi M, Ishihara O. Takotsubo cardiomyopathy after cesarean: a case report and published work review 
of pregnancy-related cases. J Obstet Gynaecol Res 2014;40(6):1534-9.

3. Sengupta S, Alsi V, Mohan V, Kaur B, Mohan JC. Unique phenotypes of typical and inverted Takotsubo cardiomyopathy in young females. Indian Heart $\mathrm{J}$ 2010;62(4):348-50.

4. Bybee KA, Kara T, Prased A, Lerman A, Barsness GW, Wright RS, et al. Systematic review: Transient left ventricular apical ballooning: A syndrome that mimics ST segment elevation myocardial infarction. An Intern Med 2004;141(11):858-65.

5. Santos Pérez A, Valero Hernández A. Miocardiopatía de Takotsubo. Claves para su diagnóstico. Gaceta Médica Espirituana 2011;13(1). Disponible en: http:// bvs.sld.cu/revistas/gme/pub/vol.13.(1)_06/p6.html.

6. Sharkey SW, Lessner JR, Zenovich AG, Maron MS, Lindberg J, Longe TF, et al. Acute and reversible cardiomyopathy provoked by stress in women from the United States. Circulation 2005;111(4):472-9

7. Akashi $\mathrm{YJ}$, Mucha H, Kida K, Ithon K, Inoue K, Kawasaki K, et al. Reversible ventricular dysfunction takotsubo cardiomyopathy. Eur J Heart Fail 2005;7(7):1171-6.

8. Citro R, Giudice R, Mirra M, Petta R, Baldi C, Bossone $\mathrm{E}$, Piscione F. Is Tako-tsubo syndrome in the postpartum period a clinical entity different from peripartum cardiomyopathy? J Cardiovasc Med (Hagerstown) 2013;14(8):568-75.

9. García E, Restrepo G, Cubides C, Múnera A, Aristizábal D. Miocardiopatía por estrés (miocardiopatía tipo takotsubo): presentación de un caso clínico y revisión de la literatura. Rev Col Cardiol 2006;13(1):31-8.

10. Nykamp D, Titak JA. Takotsubo cardiomyopathy, or broken-heart syndrome. Ann Pharmacother 2010;44(3):590-3.

11. Tsuchihashi $\mathrm{K}$, Ueshima $\mathrm{K}$, Uchida $\mathrm{T}$, et al. Transient left ventricular apical ballooning without coronary artery stenosis: a novel heart syndrome mimicking acute myocardial infarction. Angina-pectoris-myocardial infarction investigations in Japan. J Am Coll Cardiol 2001;38(1):11-18. 